\title{
Anomalous gap ratio in anisotropic superconductors: Aluminum under pressure
}

\author{
Rustem Khasanov (1) ${ }^{1, *}$ and Igor I. Mazin (1) $^{2,3}$ \\ ${ }^{1}$ Laboratory for Muon Spin Spectroscopy, Paul Scherrer Institut, CH-5232 Villigen PSI, Switzerland \\ ${ }^{2}$ Department of Physics and Astronomy, George Mason University, Fairfax, Virginia 22030, USA \\ ${ }^{3}$ Quantum Science and Engineering Center, George Mason University, Fairfax, Virginia 22030, USA
}

(Received 6 November 2020; revised 14 January 2021; accepted 26 January 2021; published 8 February 2021)

\begin{abstract}
Pressure dependence of the thermodynamic critical field $B_{c}$ in elemental aluminum was studied by means of the muon-spin rotation-relaxation technique. Pressure enhances the deviation of $B_{c}(T)$ from parabolic behavior, expected for a typical type-I superconductor, thus suggesting the weakening of the gap ratio $\langle\alpha\rangle=\langle\Delta\rangle / k_{B} T_{c}$ ( $\langle\Delta\rangle$ is the average value of the superconducting energy gap, $T_{c}$ is the transition temperature, and $k_{B}$ is the Boltzmann constant). With the pressure increase from 0.0 to $\simeq 1.6 \mathrm{GPa},\langle\alpha\rangle$ decreases almost linearly from 1.73(1) to 1.67(1). Our results imply, therefore, that in elemental aluminum, the gap ratio $\langle\alpha\rangle$ is smaller than the weak-coupled BCS prediction $\alpha_{\mathrm{BCS}} \simeq 1.764$, and it is even further reduced under pressure.
\end{abstract}

DOI: 10.1103/PhysRevB.103.L060502

In superconducting materials, the coupling strength is generally established by comparing the reduced gap (the ratio of the energy gap $\Delta$ to the transition temperature $T_{c}$ ) with the universal weak-coupling BCS number $\alpha_{\mathrm{BCS}}=\Delta / k_{B} T_{c}=$ $e^{\gamma_{E}} / \pi \simeq 1.764\left(\gamma_{E}\right.$ is the Euler constant, and $k_{B}$ is the Boltzmann constant) [1-3]. Based on such a comparison, the superconductors are divided into the strong-coupled $(\alpha \gg$ 1.764), intermediate-coupled ( $\alpha \gtrsim 1.764$ ), and weak-coupled $(\alpha \simeq 1.764)$ classes. This division has a profound physical meaning: the universal value $\alpha_{\mathrm{BCS}}$ corresponds to the temperature at which the order parameter is destroyed by its own thermal fluctuations. Any additional pair-breaking agent can only suppress $T_{c}$ and, thus, increase $\alpha$ above the $\alpha_{\mathrm{BCS}}$ value.

More accurate than BCS, the Eliashberg theory accounts for the fact that, while virtual phonons bind electrons into Cooper pairs, the real (thermal) phonons break them. In the weak-coupling limit, by definition, $T_{c}$ is exponentially smaller than the energy of the pairing bosons, so the concentration of thermal excited bosons, interacting with superconducting electrons, is exponentially low. Consequently, the reduced gap stays exponentially close to $\alpha_{\mathrm{BCS}}$. As coupling becomes stronger, the ratio of $T_{c}$ to a typical boson frequency becomes larger, and the pair-breaking effect of real bosons is not negligible anymore. With temperature, their number and, correspondingly, their pair breaking effect grows, and superconductivity becomes destroyed at $T_{c}<T_{c, \mathrm{BCS}}$. Thus, the reduced gap $\alpha=\Delta / k_{B} T_{c}>\Delta / k_{B} T_{c, \text { BCS }}=\alpha_{\mathrm{BCS}}$. By modifying the phonon spectrum, i.e., by adding very soft phonons, one can generate a paradoxical situation, where superconductivity is physically from weak coupling (the phonons generating $T_{c}$ are exponentially harder than the gap) and yet have a huge $\alpha$ ratio [4]. One may also consider two different types of bosons, one pairing and the other pair breaking, even in virtual diagrams (for instance, the former may be phonons

*rustem.khasanov@psi.ch and the latter spin fluctuations) [5], albeit, generally speaking, such a situation should also lead to an enhancement of $\alpha$.

An opposite effect, a phonon spectrum that would render $\alpha<\alpha_{\mathrm{BCS}}$, appears on the first glance impossible (barring a dramatic change of the actual phonon spectrum between $T=$ 0 and $T=T_{c}$ ). Note however that the above consideration implicitly assumes the superconducting state characterized by one single-order parameter, single in the sense that there is one number for all Cooper pairs in the system. This assumption is, of course, violated in such cases as multiband and/or anisotropic superconductivity, where the order parameters vary over the Fermi surface. In that case, $T_{c}$ is uniquely defined, but the way one collapses the function $\Delta(\mathbf{k})$ to a single average number $\langle\Delta\rangle$ is ambiguous. Interestingly, some natural definitions of $\langle\Delta\rangle$ resulting from experimental methods of measuring the order parameter may actually render the apparent $\langle\alpha\rangle=\langle\Delta\rangle / k_{B} T_{c}$ to be smaller than $\alpha_{\mathrm{BCS}}$.

Elemental aluminum is often cited as a canonical weakcoupling superconductor. Indeed, the tunneling experiments of Blackford and March [6], performed on Al polycrystalline films resulted in $\alpha=1.765(5)$, in excellent agreement with the BCS prediction. At the same time, experiments on high-quality aluminum single crystals revealed that the superconducting gap is anisotropic, i.e., it has different values along different crystallographic directions. The $\alpha$ value was found to vary from $\simeq 1.5$ to $\simeq 1.8$ [7-9], around the Fermi surface. This suggests, after a closer look, that the average reduced gap $\langle\Delta\rangle$ (of course, depending on the averaging protocol) may become smaller than the universal BCS limit $[10,11]$.

The first attempts to evaluate gap anisotropy of superconducting aluminum theoretically go back to $1971[12,13]$. These were using empirical model pseudopotentials and a spherical Fermi surface and were later somewhat improved to include a realistic Fermi surface [14]. Meza-Montes et al. [15] improved that further by taking into account the anisotropy of the Coulomb pseudopotential $\mu^{*}$, albeit they never took into account the anisotropy of the logarithmic renormalization 
of $\mu^{*}$, even though it can be argued to be more important [16]. While these calculations may be considered subpar by modern standards, they agree qualitatively with the experimental observations of Refs. [7-9] (see also a cumulative table in Ref. [17]). It is interesting to note that one of the early theoretical predictions, which is not verified experimentally so far, suggests that the gap anisotropy in aluminum should greatly increase with the applied pressure $p$ [18].

In this paper, we present experimental evidence that the reduced thermodynamic gap in elemental aluminum, defined as the gap corresponding to the thermodynamic critical field, $\langle\Delta\rangle=B_{c}(0) / \sqrt{4 \pi N_{F}(0)}\left[N_{F}(0)\right.$ is the density of states at the Fermi level and $B_{c}(0)=B_{c}(T=0)$ is the zero-temperature value of the thermodynamic critical field], is smaller than $\alpha_{\mathrm{BCS}}$ and it decreases under pressure. The analysis of the experimental $B_{c}(T, p)$ dependencies within the anisotropic gap model of Clem $[10,11]$ suggest that the mean-squared anisotropy value

$$
\left\langle a^{2}\right\rangle \equiv \frac{[\Delta(\mathbf{k})-\langle\Delta\rangle]^{2}}{\langle\Delta\rangle^{2}}
$$

changes as the pressure increases from $p=0.0$ to $\simeq 1.6 \mathrm{GPa}$, from $\left\langle a^{2}\right\rangle=0.013$ (1) to 0.035(2), which in turn, leads to a decrease of $\langle\alpha\rangle$ from 1.73(1) to 1.67(1).

The description of cylindrically shaped Al samples, piston-cylinder types of pressure cells, and transverse-field muon-spin rotation-relaxation (TF- $\mu \mathrm{SR}$ ) under pressure experiments are given in the Supplemental Material [19]. The Supplemental part includes Refs. [20-27].

The TF- $\mu$ SR measurements were performed in the intermediate state of superconducting aluminum, i.e., when the sample volume is separated into the normal state and the superconducting (Meissner) domains [2,3,28-35]. The external field $B_{\text {ex }}$ was applied perpendicular to the cylindrical axis of the sample. In this geometry, the demagnetization factor was estimated to be $n \simeq 0.42[19,20]$, so the intermediate state was set in the region of $B_{c}>B_{\text {ex }} \gtrsim 0.58 B_{c}$. The modified $B-T$-scan measuring scheme, as discussed in Refs. [33,35], was used. At each particular temperature, the measured points were reached by first decreasing $B_{\text {ex }}$ to zero and then increasing it back to the measuring ones. The $B-T$ points were taken along $\simeq 0.9,0.825,0.75$, and $0.675 B_{c}(T)$ lines by considering the $B_{c}(p, T)$ curves as they were determined in Refs. [36,37]. Such a procedure allows us to exclude effects of 'supercooling' [38-46], which were found to be particularly strong in the elemental aluminum superconductor $[38,39,45]$.

The temperature dependencies of the thermodynamic critical field $B_{c}$ at pressures ranging from $p=0.0$ to $\simeq 1.56 \mathrm{GPa}$ are presented in Fig. 1(a). The magnetic field distribution in a type-I superconductor in the intermediate state, which is probed directly by means of TF- $\mu \mathrm{SR}$, consists of two peaks corresponding to the response of the domains remaining in the Meissner state $(B=0)$ and in the intermediate state $\left(B \equiv B_{c}>B_{\text {ex }}\right)$. Consequently, in TF- $\mu$ SR experiments, the value of $B_{c}$ is directly and very precisely determined by measuring the position of the $B>B_{\text {ex }}$ peak [32-35,46-51]. The raw TF- $\mu$ SR data and details of the data analysis procedure are presented in the Supplemental Material [19]. Deviations of the $B_{c}$ vs. $T$ curves from the parabolic function $D\left(T^{2}\right)=$
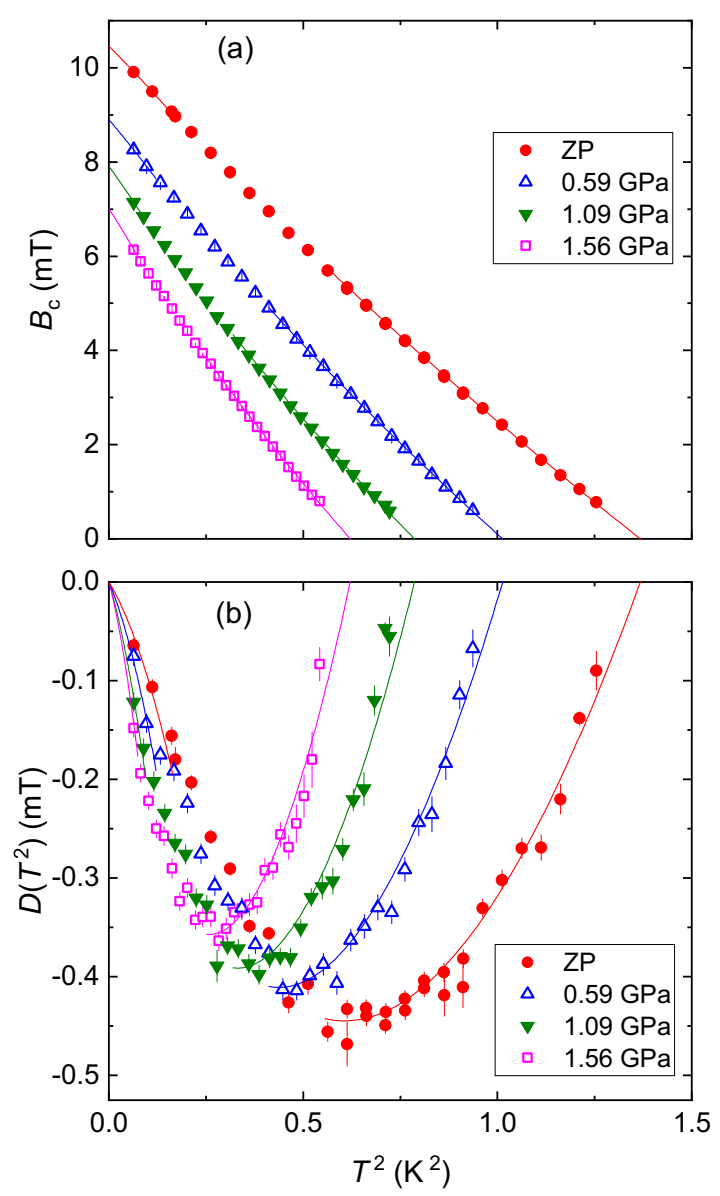

FIG. 1. (a) Temperature dependencies of the thermodynamic critical field $B_{c}$ of elemental aluminum measured at pressures $p=$ $0.0,0.59,1.09$, and $1.56 \mathrm{GPa}$. The solid lines are fits of Eqs. (2) and (4) to the low-temperature $\left[\left(T / T_{c}\right)^{2}<0.1\right]$ and the high-temperature $\left[0.5<\left(T / T_{c}\right)^{2}<1\right]$ parts of $B_{c}\left(T^{2}, p\right)$ data, respectively. (b) Temperature dependencies of the deviation function $D\left(T^{2}\right)=B_{c}\left(T^{2}\right)-$ $B_{c}(0)\left[1-\left(T / T_{c}\right)^{2}\right]$ at pressures $p=0.0,0.59,1.09$, and $1.56 \mathrm{GPa}$. The solid lines are theoretical $D\left(T^{2}, p\right)$ curves, which were reconstructed by using parameters obtained from fits of Eqs. (2) and (4) to $B_{c}\left(T^{2}, p\right)$ data (see text for details).

$B_{c}\left(T^{2}\right)-B_{c}(0)\left[1-\left(T / T_{c}\right)^{2}\right]$ are shown in Fig. 1(b). Following Refs. [10,11,52-55], the shape of $D\left(T^{2}\right)$ depends strongly on the $\Delta / k_{B} T_{c}$ ratio, and it is also expected to be sensitive to the symmetry of the superconducting energy gap.

Bearing in mind the anisotropic single-gap behavior of elemental aluminum at ambient pressure [7-9], the analysis of $B_{c}(T, p)$ dependencies was performed by means of the phenomenological model of Clem [10,11], which considers effects of gap anisotropy on fundamental thermodynamic quantities of a superconductor and is correct in the weak anisotropy limit. Strictly speaking, the analysis of temperature dependencies of various thermodynamic quantities in an anisotropic superconductor requires the exact knowledge of a momentum dependence of the superconducting energy gap [56], as well as the shape of the Fermi surface. Using the spherical Fermi surface approximation, Clem [10] obtained the analytical expressions for $B_{c}(T)$ and $D(T)$ in the 
low- and high-temperature regimes in terms of the meansquared anisotropy, Eq. (1).

Following Clem [10], in the low-temperature regime $\left(T / T_{c} \lesssim 0.3\right)$ :

$$
b_{c}(t) \simeq 1-1.057\left(1+2\left\langle a^{2}\right\rangle\right) t^{2}-0.559\left(1+4\left\langle a^{2}\right\rangle\right) t^{2},
$$

and

$$
D(t) \simeq-\left(0.057+2.11\left\langle a^{2}\right\rangle\right) t^{2}-0.559\left(1+4\left\langle a^{2}\right\rangle\right) t^{4} .
$$

Here, the notations of the reduced temperature $t=T / T_{c}$ and the reduced field $b_{c}(t)=B_{c}(T) / B_{c}(T=0)$ are used.

At higher temperatures $\left(0.7 \lesssim T / T_{c} \lesssim 1\right)$, the equations for $b_{c}(t)$ and $D(t)$ become [10]:

$$
\begin{aligned}
b_{c}(t) & \simeq 1.7367\left(1-\left\langle a^{2}\right\rangle\right)(1-t)^{2}\left[1-\left(0.273-0.908\left\langle a^{2}\right\rangle\right)\right. \\
& \left.-\left(0.0949-0.037\left\langle a^{2}\right\rangle\right)(1+t)\right],
\end{aligned}
$$

and

$$
\begin{aligned}
D(t) \simeq & -0.1317\left(1+6.6\left\langle a^{2}\right\rangle\right)\left(1-t^{2}\right) \\
& +0.0986\left(1+3\left\langle a^{2}\right\rangle\right)\left(1-t^{2}\right)^{2} \\
& +0.0287\left(1+6.15\left\langle a^{2}\right\rangle\right)\left(1-t^{2}\right)^{3} .
\end{aligned}
$$

Note that Eqs. (2) and (4) are basically the same as Eqs. (3) and (5). These two sets of equations are only different by the term $1-t^{2}$, which is introduced to obtain the deviation function $D(t)$ from the temperature evolution of the thermodynamic critical field $b_{c}(t)$ in a way $D(t)=b_{c}(t)-$ $\left(1-t^{2}\right)$. Consequently, only the $B_{c}\left(T^{2}, p\right)$ datasets were analyzed [solid lines in Fig. 1(a)]. The theoretical $D\left(T^{2}, p\right)$ curves were further reconstructed by using parameters obtained from fits of Eqs. (2) and (4) to $B_{c}\left(T^{2}, p\right)$ curves [solid lines in Fig. 1(b)]. The parameters obtained from the fits, namely the superconducting transition temperature $T_{c}$, the zero-temperature value of the thermodynamic critical field $B_{c}(0)$, and the mean-squared anisotropy $\left\langle a^{2}\right\rangle$, are summarized in Fig. 2. In addition to the results of this paper, the experimental $B_{c}(T, p=0)$ data of Harris and Mapother [55] were also reanalyzed by using the above-described approach (black asterisk symbols in Figs. 2 and 3).

From the results presented in Fig. 2, the following four important points emerge: (i) The fit parameters for ambient pressure TF- $\mu$ SR data coincide with that obtained by reanalyzing the results of magnetization data of Harris and Mapother [55]. (ii) The linear fit of $T_{c}(p)$ and $B_{c}(0, p)$ dependencies result in $d T_{c} / d p=0.248(7) \mathrm{K} / \mathrm{GPa}$ and $d B_{c}(0, p) / d p=2.20(1) \mathrm{mT} / \mathrm{GPa}$, respectively, which stays in a good agreement with the previously published data $[36,37,57,58]$. (iii) The zero-pressure value of the mean-squared anisotropy parameter was found to be $\left\langle a^{2}\right\rangle=$ $0.013(1)$. This value stays in agreement with the results of tunneling experiments of Blackford [8] and Kogure et al. [9] and the low-temperature specific heat measurements of Cheeke $e t$ al [59] reporting $\left\langle a^{2}\right\rangle \simeq 0.009-0.01$. Markowitz and Kadanoff [60] estimate $\left\langle a^{2}\right\rangle \simeq 0.011$ by analyzing measurements of the critical temperature as a function of impurity doping by Chanin et al. [61]. The theoretical predictions of Leavens and Carbotte [13,62] and Meza-Montes et al. [15] gave $\left\langle a^{2}\right\rangle=$ 0.0084 and 0.015 , respectively. (iv) With the pressure increase from $p=0.0$ to $\simeq 1.6 \mathrm{GPa}$, the mean-squared anisotropy
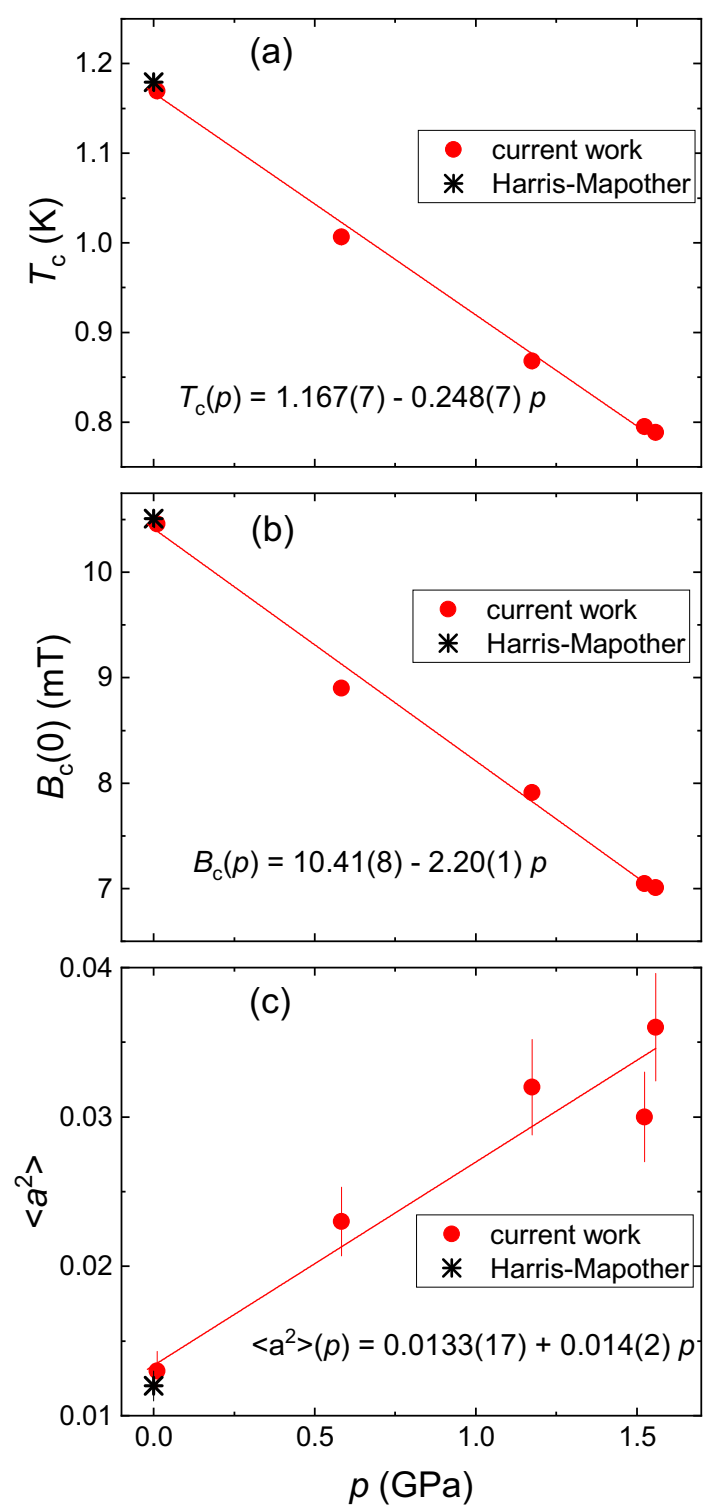

FIG. 2. Pressure dependence of (a) the superconducting transition temperature $T_{c}$, (b) the zero-temperature value of the thermodynamic critical field $B_{c}(0)$, and (c) the mean-squared anisotropy $\left\langle a^{2}\right\rangle$ of elemental aluminum. The quantities were obtained from the analysis of $B_{c}\left(T^{2}, p\right)$ data within the framework of the anisotropic model of Clem [10,11]. The circles are the data from this paper, and the asterisks are obtained by analyzing the data of Harris and Mapother [55]. The lines are linear fits.

parameter $\left\langle a^{2}\right\rangle$ almost triples, from 0.013(1) to 0.035(2) [see Fig. 2(c)]. Bearing in mind that, within the model of Clem $[10,11]$, the anisotropic gap behavior follows:

$$
\Delta(\mathbf{k})=\langle\Delta\rangle[1+a(\mathbf{k})],
$$

this suggests that the ratio between the smallest and the biggest gap $\left[\Delta(\mathbf{k})^{\max } / \Delta(\mathbf{k})^{\mathrm{min}}\right]$ increases from $\simeq 1.25$ at ambient pressure to $\simeq 2.2$ at $p \simeq 1.6 \mathrm{GPa}$. Here, the simplest case (the two-gap case) with $a(\mathbf{k})= \pm a_{0}$ and with the equal weight of $\langle\Delta\rangle\left(1 \pm a_{0}\right)$ gaps was considered.

As the next step, we are going to compare the results of this paper with the calculations of Leavens and Carbotte [18]. 

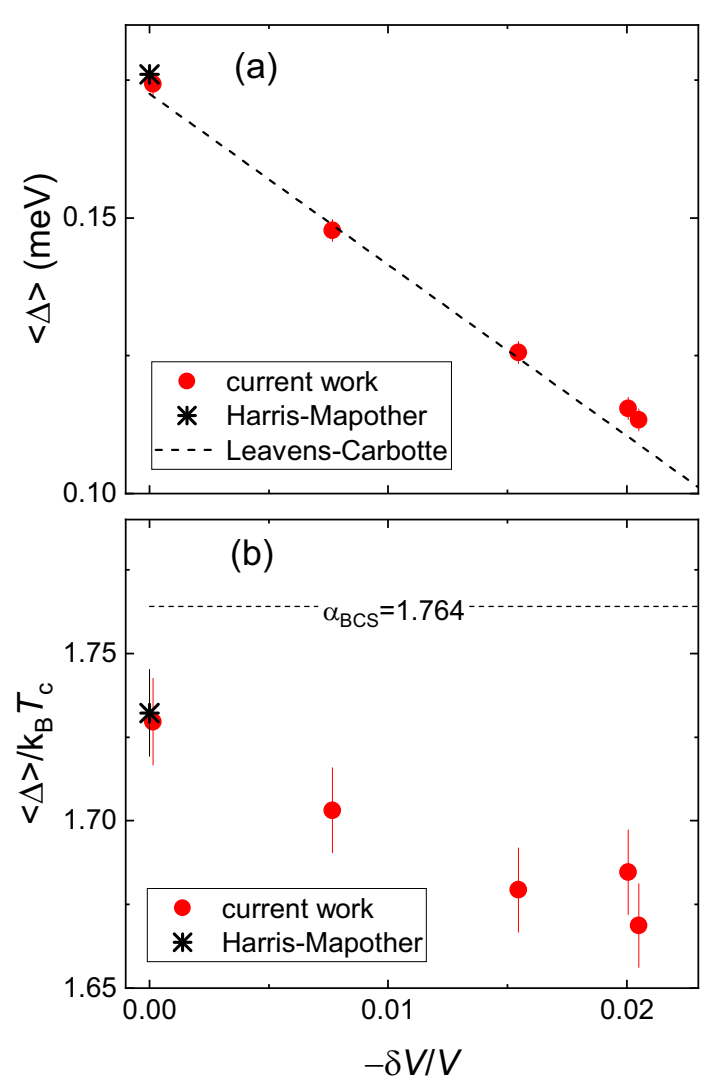

FIG. 3. (a) Dependence of the averaged gap value $\langle\Delta\rangle$ on the relative volume change $-\delta V / V$. The dashed line is the theory prediction of Leavens and Carbotte [18]. (b) Dependence of $\langle\alpha\rangle=\langle\Delta\rangle / k_{B} T_{c}$ on $-\delta V / V$. The dashed line is the weak-coupling BCS number $\alpha_{\mathrm{BCS}} \simeq 1.764$. The closed circles correspond to the data obtained within this paper. The black asterisks are parameters obtained after analyzing the data of Harris and Mapother [55].

Figure 3(a) shows the average gap value $\langle\Delta\rangle$ as a function of the reduced volume $-\delta V / V$. From the present data, the value of $\langle\Delta\rangle$ was calculated via [10]

$$
\frac{\langle\Delta\rangle}{k_{B} T_{c}}=1.764\left(1-\frac{3}{2}\left\langle a^{2}\right\rangle\right),
$$

and the relative volume change was obtained as $-\delta V / V=K p$ ( $K \simeq 76 \mathrm{GPa}$ is the bulk modulus of aluminum, Ref. [63]). Figure 3(a) points to very good agreement between the theory and the experiment.

Figure 3(b) shows the dependence of $\langle\alpha\rangle=\langle\Delta\rangle / k_{B} T_{c}$ on the relative volume change $-\delta V / V$. The results presented in the graph are twofold: (i) All the experimental points lie below the weak-coupling BCS value $\alpha_{\mathrm{BCS}} \simeq 1.764$. As already stated in the introduction, it is not possible for isotropic superconductor to have the coupling strength parameter smaller than the universal BCS number $\alpha \nless \alpha_{\mathrm{BCS}}$. This implies that the superconducting energy gap in elemental aluminum must be anisotropic, and it remains anisotropic within the full pres- sure range studied here. Only in this particular case, $\langle\alpha\rangle$ of aluminum may become smaller than $\alpha_{\text {BCS }}$. (ii) The sample compression leads to continuous decrease of $\langle\alpha\rangle$. Our experiments for aluminum indicate that the effect is quite large. Here, $\langle\alpha\rangle$ decreases by slightly more than $5 \%$ for a relative volume change of $\simeq 2 \%$.

Leavens and Carbotte [18] provided a simple explanation for the pressure dependence of the superconducting gap anisotropy. Following Refs. [10,18], the dominant source of the gap anisotropy is the anisotropy of the phonon-mediated electron-electron interaction. When the metal is subjected to a hydrostatic pressure, the Fermi sphere and the Brillouin zone scale together, so that the shape of the complicated surface generated for the initial point $(\theta, \phi)$ does not change. It is expected, therefore, that even though the absolute value of the electron-phonon coupling constant $\lambda_{\text {e-p }}$ changes as a function of pressure, the anisotropy of the phonon-induced electron-electron interaction remains unaltered. Bearing in mind that the Coulomb pseudopotential parameter $\mu^{*}$ changes very slowly with pressure as compared with the phononmediated interaction, the anisotropy in the total interaction increases. This said, full density functional theory calculations of the anisotropic Eliashberg function and k-dependent order parameter in $\mathrm{Al}$ are highly desirable.

The above arguments would also imply that the smaller the ratio of the electron-phonon interaction to the strength of the Coulomb interaction, the faster the increase of the energy gap anisotropy with pressure. In strong coupling superconductors, $\lambda_{\text {e-p }}$ is about 10 times higher than $\mu^{*} \sim 0.1-0.15$, which is only weakly dependent on the material. Note that the size of $\mu^{*}$ is fixed by the Tolmachev logarithm [64] $\mu^{*} \approx \mu /[1+$ $\mu \ln \left(\theta_{D} / T_{c}\right) \approx 1 / \ln \left(\theta_{D} / T_{c}\right)$. It might be expected, therefore, that the variation of the electron-phonon interaction as a function of pressure in strongly coupled superconductors will lead to a small pressure effect on the gap anisotropy. In weakly coupled superconductors, however, $\lambda_{\text {e-p }}$ is smaller, and the pressure dependence of the superconducting gap anisotropy is expected to be more pronounced. This is definitively the case for aluminum, where $\lambda_{\text {e-p }} \sim 4 \mu^{*}$.

To conclude, the pressure dependence of the thermodynamic critical field $B_{c}$ in elemental aluminum was studied by means of the muon-spin rotation-relaxation. Pressure was found to enhance the deviation of $B_{c}(T)$ from the parabolic behavior, thus suggesting the weakening of the reduced gap $\langle\alpha\rangle=\langle\Delta\rangle / k_{B} T_{c}$. The analysis of the experimental data within the anisotropic gap model of Clem [10,11] suggests that, with the pressure increase from 0.0 to $\simeq 1.6 \mathrm{GPa}$, the mean-squared anisotropy value $\left\langle a^{2}\right\rangle$ changes from $0.013(1)$ to $0.035(2)$, which in turn, leads to a change of $\langle\alpha\rangle$ from 1.73(1) to $1.67(1)$.

This paper was performed at the Swiss Muon Source $(\mathrm{S} \mu \mathrm{S})$, Paul Scherrer Institute (PSI, Switzerland). RK acknowledges the technical support of Matthias Elender, Ritu Gupta, and Debarchan Das. The authors acknowledge helpful discussions with Rafael Fernandes and Douglas Finnemore.
[1] J. Bardeen, L. N. Cooper, and J. R. Schrieffer, Phys. Rev. 106, 162 (1957).
[2] M. Tinkham, Introduction to Superconductivity (Krieger Publishing Company, Malabar, 1975). 
[3] C. Poole, H. Farach, R. Creswick, and R. Prozorov, Superconductivity, 3rd ed. (Elsevier, Amsterdam, 2014).

[4] I. I. Mazin, O. V. Dolgov, A. Golubov, and S. V. Shulga, Phys. Rev. B 47, 538 (1993).

[5] O. V. Dolgov, I. I. Mazin, A. A. Golubov, S. Y. Savrasov, and E. G. Maksimov, Phys. Rev. Lett. 95, 257003 (2005)

[6] B. L. Blackford and R. H. March, Can. J. Phys. 46, 141 (1968).

[7] G. L. Wells, J. E. Jackson, and E. N. Mitchell, Phys. Rev. B 1, 3636 (1970).

[8] B. L. Blackford, J. Low Temp. Phys. 23, 43 (1976).

[9] Y. Kogure, N. Takeuchi, Y. Hiki, K. Mizuno, and T. Kino, J. Phys. Soc. Jpn. 54, 3506 (1985).

[10] J. R. Clem, Ann. Phys. (NY) 40, 268 (1966).

[11] J. R. Clem, Phys. Rev. 153, 449 (1967).

[12] R. C. Dynes and J. P. Carbotte, Physica 55, 462 (1971).

[13] C. R. Leavens and J. P. Carbotte, Ann. Phys. 70, 338 (1972).

[14] H. K. Leung, J. P. Carbotte, and C. R. Leavens, J. Low Temp. Phys. 24, 25 (1976).

[15] L. Meza-Montes, R. A. Brito-Orta, and R. Baquero, J. Low Temp. Phys. 70, 413 (1988).

[16] I. I. Mazin, O. K. Andersen, O. Jepsen, A. A. Golubov, O. V. Dolgov, and J. Kortus, Phys. Rev. B 69, 056501 (2004).

[17] X. H. Zheng and D. G. Walmsley, Physica C 534, 19 (2017), Table 3.

[18] C. R. Leavens and J. P. Carbotte, Can. J. Phys. 50, 2568 (1972).

[19] See Supplemental Material at https://link.aps.org/supplemental/ 10.1103/PhysRevB.103.L060502 for a description of the Al samples, the piston-cylinder type of pressure cells, and transverse-field muon-spin rotation-relaxation (TF- $\mu \mathrm{SR}$ ) under pressure experiments. The Supplemental Material includes Refs. [20-27].

[20] R. Prozorov and V. G. Kogan, Phys. Rev. Applied 10, 014030 (2018).

[21] R. Khasanov, Z. Guguchia, A. Maisuradze, D. Andreica, M. Elender, A. Raselli, Z. Shermadini, T. Goko, E. Morenzoni, and A. Amato, High Press. Res. 36, 140 (2016).

[22] Z. Shermadini, R. Khasanov, M. Elender, G. Simutis, Z. Guguchia, K. V. Kamenev, and A. Amato, High Press. Res. 37, 449 (2017).

[23] K. Murata, H. Yoshino, H. O. Yadev, Y. Honda, and N. Shirakawa, Rev. Sci. Instrum. 68, 2490 (1997).

[24] M. I. Eremets, High Pressure Experimental Methods (Oxford University Press, London, 1996).

[25] S. Klotz, Techniques in High Pressure Neutron Scattering (CRC Press, Boca Raton, 2012).

[26] A. Suter and B. M. Wojek, Phys. Procedia 30, 69 (2012).

[27] A. Yaouanc and P. Dalmas de Réotier, Muon Spin Rotation, Relaxation and Resonance: Applications to Condensed Matter (Oxford University Press, Oxford, 2011).

[28] C. Kittel, Introduction to Solid State Physics, 7th ed. (Wiley India Pvt. Limited, New-York, 2007).

[29] P. G. de Gennes, Superconductivity of Metals and Alloys (Benjamin, New York, 1966).

[30] R. Prozorov, Phys. Rev. Lett. 98, 257001 (2007).

[31] R. Prozorov, A. F. Fidler, J. R. Hoberg, and P. C. Canfield, Nat. Phys. 4, 327 (2008).

[32] R. Khasanov, M. M. Radonjić, H. Luetkens, E. Morenzoni, G. Simutis, S. Schönecker, W. H. Appelt, A. Östlin, L. Chioncel, and A. Amato, Phys. Rev. B 99, 174506 (2019).
[33] R. Karl, F. Burri, A. Amato, M. Donegà, S. Gvasaliya, H. Luetkens, E. Morenzoni, and R. Khasanov, Phys. Rev. B 99, 184515 (2019).

[34] R. Khasanov, H. Luetkens, A. Amato, and E. Morenzoni, Phys. Rev. B 101, 054504 (2020).

[35] R. Khasanov, R. Gupta, D. Das, A. Amon, A. Leithe-Jasper, and E. Svanidze, Phys. Rev. Research 2, 023142 (2020).

[36] R. I. Boughton, J. L. Olsen, and C. Palmy, in Progress in LowTemperature Physics, edited by C. J. Gorter, (Noorth-Holland Publishing Company, Amsterdam, 1970).

[37] C. Palmy, R. Flach, and P. de Trey, Physica 55, 663 (1971).

[38] J. F. Cochran, D. E. Mapother, and R. E. Mould, Phys. Rev. 103, 1657 (1956).

[39] T. E. Faber, Proc. Roy. Soc. (London) A 241, 531 (1957).

[40] D. Saint-James and P. G. de Gennes, Phys. Lett. 7, 306 (1963).

[41] J. P. McEvoy, D. P. Jones, and J. G. Park, Solid St. Commun. 5, 641 (1967).

[42] J. G. Park, Solid St. Commun. 5, 645 (1967).

[43] J. Feder, Solid St. Commun. 5, 299 (1967).

[44] F. W. Smith and M. Cardona, Solid St. Commun. 6, 37 (1968).

[45] F. W. Smith, A. Baratoff, and M. Cardona, Phys. Kondens. Mater. 12, 145 (1970).

[46] V. S. Egorov, G. Solt, C. Baines, D. Herlach, and U. Zimmermann, Phys. Rev. B 64, 024524 (2001).

[47] M. Gladisch, D. Herlach, H. Metz, H. Orth, G. zu Putlitz, A. Seeger, H. Teichler, W. Wahl, and W. Wigand, Hyperfine Interact. 6, 109 (1979).

[48] V. G. Grebinnik, I. I. Gurevich, V. A. Zhukov, A. I. Klimov, L. A. Levina, V. N. Maiorov, A. P. Manych, E. V. Mel'nikov, B. A. Nikol'skii, A. V. Pirogov, A. N. Ponomarev, V. S. Roganov, V. I. Selivanov, and V. A. Suetin, Zh. Eksp. Teor. Fiz. 79, 518 (1980) [Sov. Phys. JETP 52, 261 (1980)].

[49] H. Leng, J.-C. Orain, A. Amato, Y. K. Huang, and A. de Visser, Phys. Rev. B 100, 224501 (2019).

[50] J. Beare, M. Nugent, M. N. Wilson, Y. Cai, T. J. S. Munsie, A. Amon, A. Leithe-Jasper, Z. Gong, S. L. Guo, Z. Guguchia, Y. Grin, Y. J. Uemura, E. Svanidze, and G. M. Luke, Phys. Rev. B 99, 134510 (2019).

[51] V. Kozhevnikov, A. Suter, T. Prokscha, and C. Van Haesendonck, J. Supercond. Nov. Magn. 33, 3361 (2020).

[52] H. Padamsee, J. E. Neighbor, and C. A. Shiffman, J. Low Temp. Phys. 12, 387 (1973).

[53] D. C. Johnston, Supercond. Sci. Technol. 26, 115011 (2013).

[54] R. Khasanov, R. Gupta, D. Das, A. Leithe-Jasper, and E. Svanidze, Phys. Rev. B 102, 014514 (2020).

[55] E. P. Harris and D. E. Mapother, Phys. Rev. 165, 522 (1968).

[56] V. G. Kogan and R. Prozorov, arXiv:2011.12412.

[57] M. Levy and J. L. Olsen, Solid St. Commun. 2, 137 (1964).

[58] D. U. Gubser and A. W. Webb, Phys. Rev. Lett. 35, 104 (1975).

[59] J. D. N. Cheeke and E. Ducla-Soares, J. Low Temp. Phys. 11, 687 (1973).

[60] D. Markowitz and L. P. Kadanoff, Phys. Rev. 131, 563 (1963).

[61] G. Chanin, E. A. Lynton, and B. Serin, Phys. Rev. 114, 719 (1959).

[62] C. R. Leavens and J. P. Carbotte, Can. J. Phys. 49, 724 (1971).

[63] https://periodictable.com/Properties/A/BulkModulus.al.html.

[64] V. V. Tolmachev, Dokl. Akad. Nauk SSSR 140, 563 (1961). 